\title{
On MIMO Cognitive Radios with Antenna Selection
}

\author{
Muhammad Fainan Hanif and Peter J. Smith \\ Department of Electrical and Computer Engineering \\ University of Canterbury, Christchurch \\ New Zealand \\ Email: \\ mfh21@student.canterbury.ac.nz \\ p.smith@elec.canterbury.ac.nz
}

\begin{abstract}
With the ever increasing interest in multiple-input multiple-output (MIMO) cognitive radio (CR) systems, reducing the costs associated with RF-chains at the radio front end becomes a very important factor. In this paper, we propose two solutions to the problem of joint transmit-receive antenna selection with the objective of maximizing data rates and satisfying interference constraints at the primary user (PU) receiver. In the first method we approximate the original non-convex optimization problem with an iterative way of solving a series of smaller convex problems. Then we present a novel, norm-based transmit receive antenna selection technique that simultaneously improves throughput while maintaining the $P U$ interference constraints. We show that this simple approach yields near optimal results with massive complexity reductions. In addition to making a performance comparison between the proposed approaches and the optimal exhaustive search approach, we establish that antenna selection is a promising option for future MIMO CR devices.
\end{abstract}

\section{INTRODUCTION}

Cognitive radios (CRs) are being considered as a promising solution to the perceived scarcity of the radio frequency (RF) spectrum [1]. The concept of multiple-input multiple-output (MIMO) CR systems has triggered a lot of interest in the research community [2]-[5]. This is because multiple antennas can be used to provide the traditional rate benefits in addition to having a role in effective interference control at the primary user (PU) receiver (RX) [3]. However, along with the gains, comes hardware complexity at the radio front end owing to the requirement of having costly RF chains (consisting of low noise amplifiers, downconverters and analog-to-digital converters) that scale with the number of antennas being used.

It is well known that antenna selection techniques present an elegant solution to such problems, see, for example, [6]-[8] and the references therein. Recent work in this area includes [9] where transmit antenna selection was considered for a multiple input single output (MISO) CR operating in the presence of a single input single output (SISO) PU. In this paper, we consider the problem of joint selection of transmit/receive antennas in a MIMO CR device. The selection procedure aims to maximize the achievable rate while satisfying any interference constraints due to the PU RX(s) (either equipped with a single antenna or multiple antennas) operating in the vicinity. The work in [9] has similarities in that the signal-to-interferenceplus-noise ratio (SINR) results in [9] can be transformed to rates, they also impose interference constraints and consider a range of selection strategies including norm based approaches. In contrast, the approach in [9] is analytically based whereas our techniques are more focused on optimization. Furthermore, in this paper we include the effects of interference from the PU to the CR and power control at the CR transmitter (TX). After formulating the antenna selection problem in the context of CR networks, we propose two solutions in addition to the brute force, optimal full search method. A comparison of the proposed algorithms, based on their performance, is also presented. Our key contributions are as follows:

- We provide an approximate solution to the original nonconvex optimization problem based on iteratively solving a series of convex problems. The results are found to be stable and in close agreement with those obtained from the optimal search.

- We present a norm-based heuristic that performs transmit and receive antenna selection, to increase the rate while satisfying the interference constraints. The heuristic has massively reduced computational complexity and gives very accurate results when compared with the optimal search.

- We demonstrate that even under interference constraints, the CR device is still able to achieve substantial rate gains due to selection (especially when the strength of the CR-PU interference channel is lower than that of the CR-CR channel) and thus retain the traditional spatial multiplexing benefit of MIMO systems.

The rest of the paper is organized as follows: Section II describes the system model and Section III presents the analytical framework. Finally, results and conclusions are given in Section IV and Section V respectively.

Notation: Boldface uppercase is used for matrices and boldface lowercase for vectors. $\operatorname{det}(),. \operatorname{Tr}($.$) and (.)^{\dagger}$ denote the determinant, trace and the conjugate transpose operators respectively. $\mathbf{I}_{M}$ denotes an $M \times M$ identity matrix and $\mathbb{E}[$. represents the statistical expectation operator. $\mathbb{C}^{x \times y}$ denotes the space of $x \times y$ matrices with complex entries. $\mathcal{C N}(\mathbf{0}, \boldsymbol{\Gamma})$ represents the distribution of a zero mean circularly symmetric complex Gaussian (ZMCSCG) vector with covariance matrix $\Gamma$. $\min ($.$) and \|.\|_{2}$ denote the minimum and $l_{2}$ norm operators respectively. $\mathbf{M}(i,:)$ and $\mathbf{M}(:, j)$ are used to represent the $i$ th row and $j$ th column of a matrix, $\mathbf{M}$, respectively. $\operatorname{Diag}($. gives the diagonal elements of a matrix. Finally, $\operatorname{diag}([\mathbf{x}], 0)$ 


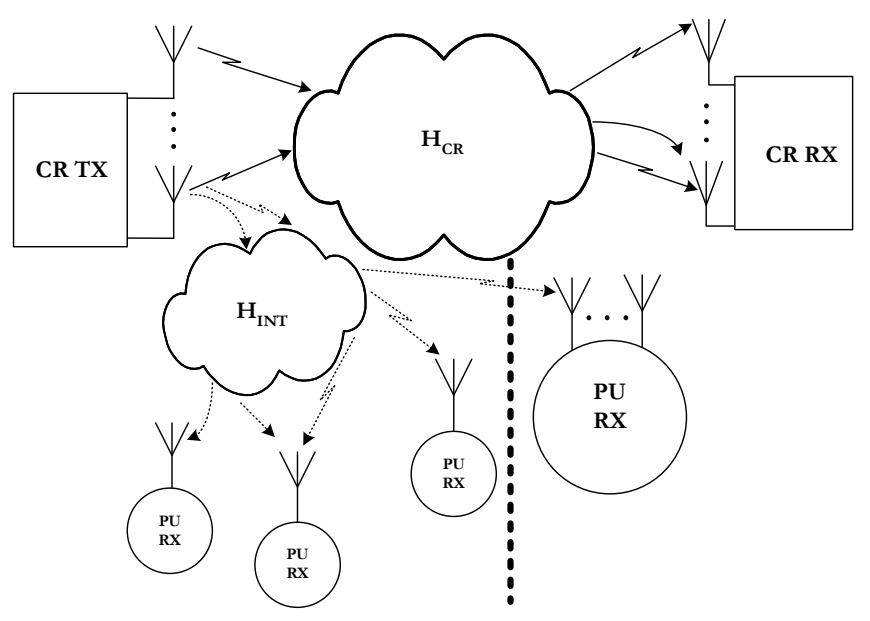

Fig. 1. System model. The vertical dotted line indicates that the multiple antenna and single antenna PU systems are considered separately.

represents a diagonal matrix with vector $\mathrm{x}$ along the diagonal and $\mathbf{M} \succeq \mathbf{0}$ indicates that $\mathbf{M}$ is a positive semidefinite matrix.

\section{SYSTEM MODEL}

The proposed system model is shown in Fig. 1. We assume that the CR TX and RX are equipped with $N_{C R}$ and $M_{C R}$ antennas respectively. The incumbent PU has $N_{P U}$ transmit (not shown in Fig. 1) and $M_{P U}$ receive antennas in the case of a single MIMO PU. We also consider the case of multiple single receive antenna PUs operating in the vicinity of the CR system. In this case $M_{P U}$ is the number of PUs. These scenarios are referred to as single user (SU) and multiuser (MU) respectively. The channels between all nodes are assumed to experience frequency flat Rayleigh fading. The signal at the $\mathrm{CR} \mathrm{RX}$ is:

$$
\mathbf{y}_{\mathrm{CR}}(n)=\mathbf{H}_{\mathrm{CR}} \mathbf{x}(n)+\mathbf{i}(n)+\mathbf{z}(n),
$$

where $\mathbf{H}_{\mathrm{CR}} \in \mathbb{C}^{M_{C R} \times N_{C R}}$ is the channel gain matrix with ZMCSCG entries, $\mathbf{y}_{\mathrm{CR}}(n)$ and $\mathbf{x}(n)$ are the received and the transmitted signal vectors respectively, $\mathbf{z}(n) \sim \mathcal{C N}\left(0, \mathbf{I}_{M_{C R}}\right)$, $\mathbf{i}(n)$ is the interference received from the PU TX(s) and the index $n$ represents the $n$th time sample. Further, the transmit covariance matrix of the $\mathrm{CR}$ user is denoted by $\mathrm{Q}_{\mathrm{CR}}=$ $\mathbb{E}\left[\mathbf{x}(n) \mathbf{x}(n)^{\dagger}\right]$. We assume that the total $\mathrm{CR}$ transmit power is limited to $P_{C R}$ i.e., $\operatorname{Tr}\left(\mathbf{Q}_{\mathrm{CR}}\right) \leq P_{C R}$. Since a normalized $\mathrm{CR}-\mathrm{CR}$ channel is considered, we have $\mathbb{E}\left[\left|\left(\mathbf{H}_{\mathrm{CR}}\right)_{i j}\right|^{2}\right]=1$ and the receive $\mathrm{SNR}$ at the $\mathrm{CR} \mathrm{RX}$ is given by $\mathrm{SNR}=P_{C R}$. The PU system has an SNR that is denoted $\mathrm{SNR}_{\mathrm{PU}}$. The covariance matrix of the interference-plus-noise is defined by $\mathbf{K}=\mathbb{E}\left[\mathbf{i}(n) \mathbf{i}(n)^{\dagger}+\mathbf{z}(n) \mathbf{z}(n)^{\dagger}\right]=\mathbf{I}_{M_{C R}}+\mathbf{H}_{\mathrm{PC}} \mathbf{H}_{\mathrm{PC}}^{\dagger}$ where we have assumed the PU TX(s) have channel $\mathbf{H}_{\mathrm{PC}}$ to the $\mathrm{CR}$ $\mathrm{RX}$ and transmit unit power uncorrelated signals. For the CR to PU interference channel, $\mathbf{H}_{\mathrm{INT}}$ (see Fig. 1), we assume $\mathbb{E}\left[\left|\left(\mathbf{H}_{\mathrm{INT}}\right)_{i j}\right|^{2}\right]=\Gamma_{i}$ where $\Gamma_{i}=\alpha$ for the SU case and $\Gamma_{i}=\alpha^{i}, i=1, \ldots, M_{P U}$ for the MU case. The constant $\alpha$ gives the strength of the dominant interference channel relative to the CR-CR channel and in the MU case it is assumed that the CR interference power decays exponentially across the PU receivers [10]. Perfect channel state information (CSI) $\left(\mathbf{H}_{\mathrm{CR}}\right.$,
$\mathbf{H}_{\mathrm{INT}}$ and $\mathbf{H}_{\mathrm{PC}}$ ) is assumed to be available at both the CR TX and CR RX for antenna selection purposes. For satisfactory operation of the incumbent PU in the presence of the CR TX, the interference seen at the PU RX should not exceed a particular threshold. This gives rise to two types of interference constraints depending on whether the PU is SU or MU. In the SU case, the interference constraint can be written as:

$\sum_{j=1}^{M_{P U}} \mathbf{H}_{\mathrm{INT}}(j,:) \mathbf{Q}_{\mathrm{CR}} \mathbf{H}_{\mathrm{INT}}(j,:)^{\dagger} \leq \Omega \Rightarrow \operatorname{Tr}\left(\mathbf{H}_{\mathrm{INT}} \mathbf{Q}_{\mathrm{CR}} \mathbf{H}_{\mathrm{INT}}^{\dagger}\right) \leq \Omega$

where $\mathbf{H}_{\mathrm{INT}}(j,:) \in \mathbb{C}^{1 \times N_{C R}}$ represents the channel from the CR TX to the $j$ th receive antenna of the PU RX and $\Omega$ is the maximum tolerable total interference at the PU RX. For the MU case the interference constraint is given by:

$$
\mathbf{H}_{\mathrm{INT}}(j,:) \mathbf{Q}_{\mathrm{CR}} \mathbf{H}_{\mathrm{INT}}(j,:)^{\dagger} \leq \omega_{j} \quad j=1,2, \ldots, M_{P U}
$$

where $\omega_{j}$ is the interference constraint for the $j$ th user. For the sake of notational convenience (3) is rewritten as $\operatorname{Diag}\left(\mathbf{H}_{\mathrm{INT}} \mathbf{Q}_{\mathrm{CR}} \mathbf{H}_{\mathrm{INT}}^{\dagger}\right) \leq\left(\omega_{1}, \ldots, \omega_{M_{P U}}\right)$, where the inequality is to be interpreted elementwise.

\section{AnAlytical FrameWORK}

We aim at performing joint transmit-receive antenna selection at the $\mathrm{CR}$ such that the $\mathrm{CR}$ rates are maximized subject to the interference constraints. The achievable rates of the $\mathrm{CR}$ system using all antennas are determined using [11]:

$$
R\left(\mathbf{H}_{\mathrm{CR}}, \mathbf{Q}_{\mathrm{CR}}\right)=\log _{2} \operatorname{det}\left(\mathbf{I}_{M_{C R}}+\mathbf{H}_{\mathrm{CR}} \mathbf{Q}_{\mathrm{CR}} \mathbf{H}_{\mathrm{CR}}^{\dagger} \mathbf{K}^{-1}\right) \text {. }
$$

Similar to the approach of [12], we define diagonal selection matrices $\mathbf{S}_{1}, \mathbf{S}_{2}$ of dimensions $M_{C R} \times M_{C R}$ and $N_{C R} \times N_{C R}$ respectively with binary diagonal entries. Specifically,

$$
\left(\mathbf{S}_{i}\right)_{k k}= \begin{cases}1 & \text { if the } k \text { th antenna element is selected } \\ 0 & \text { otherwise }\end{cases}
$$

where $i=1,2$. The diagonal entries of $\mathbf{S}_{1}, \mathbf{S}_{2}$ give the indices of the antennas selected on the CR RX and the CR TX side respectively. Hence, if $m_{c r} \leq M_{C R}$ receive antennas and $n_{c r} \leq N_{C R}$ transmit antennas are selected we obtain a new CR channel matrix $\overline{\mathbf{H}}_{\mathrm{CR}}$ with $M_{C R}-m_{c r}$ rows and $N_{C R}-n_{c r}$ columns in $\mathbf{H}_{\mathrm{CR}}$ replaced with zeros. Thus, the rate expression becomes:

$$
R\left(\overline{\mathbf{H}}_{\mathrm{CR}}, \overline{\mathbf{Q}}_{\mathrm{CR}}\right)=\log _{2} \operatorname{det}\left(\mathbf{I}_{M_{C R}}+\overline{\mathbf{H}}_{\mathrm{CR}} \overline{\mathbf{Q}}_{\mathrm{CR}} \overline{\mathbf{H}}_{\mathrm{CR}}^{\dagger}\right)
$$

where $\overline{\mathbf{H}}_{\mathrm{CR}}=\overline{\mathbf{K}}^{-1 / 2} \mathbf{S}_{1} \mathbf{H}_{\mathrm{CR}} \mathbf{S}_{2}$ and $\overline{\mathbf{K}}$ and $\overline{\mathbf{Q}}_{\mathrm{CR}}$ are defined as follows. With the selected receive antennas we have reduced $m_{c r} \times 1$ interference and noise vectors which give a new interference and noise covariance matrix, $\mathbf{K}_{\text {red }}$, of dimension $m_{c r} \times m_{c r}$. This matrix is inflated to form $\overline{\mathbf{K}}$, an $M_{C R} \times M_{C R}$ matrix, by adding rows and columns of zeros corresponding to the receive antennas not selected. Similarly, a reduced $\mathbf{Q}_{\text {red }}$ matrix $\left(n_{c r} \times n_{c r}\right)$ is formed corresponding to the transmit antennas selected which is inflated to form $\overline{\mathbf{Q}}\left(N_{C R} \times N_{C R}\right)$ by inserting rows and columns of zeros corresponding to transmit antennas not selected. With this notation, the problem of joint transmit-receive antenna selection together with CR power 
allocation can be mathematically cast in the SU case as:

$$
\begin{aligned}
\text { P1: maximize } & \log _{2} \operatorname{det}\left(\mathbf{I}_{M_{C R}}+\overline{\mathbf{K}}^{-1 / 2} \mathbf{S}_{1} \mathbf{H}_{\mathrm{CR}} \mathbf{S}_{2} \mathbf{Q}_{\mathrm{CR}} \mathbf{S}_{2}^{\dagger}\right. \\
& \left.\times \mathbf{H}_{\mathrm{CR}}^{\dagger} \mathbf{S}_{1}^{\dagger} \overline{\mathbf{K}}^{-1 / 2}\right) \\
\text { subject to } & \left(\mathbf{S}_{i}\right)_{j j} \in\{1,0\}, j=1, \ldots, M_{C R} \text { if } i=1 \\
& \text { and } j=1, \ldots, N_{C R} \text { if } i=2 \\
& \operatorname{Tr}\left(\mathbf{Q}_{\mathrm{CR}}\right) \leq P_{C R}, \operatorname{Tr}\left(\mathbf{S}_{1}\right)=m_{c r}, \operatorname{Tr}\left(\mathbf{S}_{2}\right)=n_{c r} \\
& \operatorname{Tr}\left(\mathbf{H}_{\mathrm{INT}} \mathbf{S}_{2} \mathbf{Q}_{\mathrm{CR}} \mathbf{S}_{2}^{\dagger} \mathbf{H}_{\mathrm{INT}}^{\dagger}\right) \leq \Omega, \quad \mathbf{Q}_{\mathrm{CR}} \succeq \mathbf{0} \\
\text { variables } & \mathbf{S}_{1}, \mathbf{S}_{2}, \mathbf{Q}_{\mathrm{CR}}
\end{aligned}
$$

We note that in $\mathbf{P 1}$ we have slightly modified the interference constraint in (2) to represent the effective interference seen at the PU RX due to the selected CR transmit antennas only. The above problem can be written for the MU case by simply replacing the interference constraint with (3) and incorporating the column selection matrix $\mathbf{S}_{2}$. In the log-determinant of P1 it is important to note that the effect of the interference and noise covariance matrix is separated from the channel, $\mathbf{H}_{\mathrm{CR}}$, by the selection matrix, $\mathbf{S}_{1}$. In systems with a fixed number of antennas it is common to construct an equivalent channel which, in (4), would correspond to $\mathbf{K}^{-1 / 2} \mathbf{H}_{\mathrm{CR}}$. Then, the analysis proceeds simply by considering $\mathbf{K}^{-1 / 2} \mathbf{H}_{\mathrm{CR}}$ rather than $\mathbf{H}_{\mathrm{CR}}$. In our situation the interference and noise covariance matrix changes for every $\mathbf{S}_{1}$ and so we cannot select rows or columns of the equivalent channel. Instead, selection from $\mathbf{H}_{\mathrm{CR}}$ is performed first, followed by the use of the corresponding $\overline{\mathbf{K}}^{-1 / 2}$ and then maximizing the resultant expression subject to the constraints shown. This makes the problem much more difficult as discussed in Sec. III-B.

\section{A. Exhaustive Search}

A straightforward way to solve $\mathbf{P 1}$ is to perform an exhaustive search (ES) over all possible combinations of antenna elements and only optimize over $\mathbf{Q}_{\mathrm{CR}}$. Hence, ES amounts to optimizing $\mathbf{Q}_{\mathrm{CR}},\left(\begin{array}{c}N_{C R} \\ n_{c r}\end{array}\right) \times\left(\begin{array}{c}M_{C R} \\ m_{c r}\end{array}\right)$ times subject to interference and total transmit power constraints. Each single optimization over $\mathbf{Q}_{\mathrm{CR}}$ is a convex problem that can be efficiently solved in polynomial time using interior-point methods [13]. However, the need to iterate through all possible combinations gives a complexity which explodes for higher dimensional systems. Throughout the paper we obtain numerical solutions to the optimization problems using CVX [14].

\section{B. Convex Approximation}

Problem P1 is highly non-convex and can be classified as an example of an integer programming problem, since two of the variables $\mathbf{S}_{1}$ and $\mathbf{S}_{2}$ are binary [15]. The nonconvexity of the problem arises due to the nature of the objective function, interference and the binary constraints. Further, the binary variables render the problem NP-hard [12]. In order to produce a more computationally efficient approach we modify the problem in the following ways. Firstly, the binary structure of $\mathbf{S}_{1}$ and $\mathbf{S}_{2}$ can be relaxed so that the antenna selection variables take on values in the interval 0 to 1. This makes the problem far easier to solve than the original integer program [13]. In addition to this, we also transform the interference constraint in $\mathbf{P 1}$ from being applicable over the selected transmit antennas to all CR transmit antennas. This yields a simpler constraint for optimization and also corresponds to the relaxation approach where the selection matrices are fractional rather than binary. Finally, we note that in this approach the effect of the $\mathbf{K}$ matrix cannot be included and $\mathbf{Q}_{\mathrm{CR}}$ is restricted to a diagonal power allocation matrix. These limitations are discussed below. With these changes, P1 can be rewritten as:

$$
\begin{aligned}
\text { P2: maximize } & \log _{2} \operatorname{det}\left(\mathbf{I}_{M_{C R}}+\mathbf{S}_{1} \mathbf{H}_{\mathrm{CR}} \mathbf{S}_{2} \mathbf{Q}_{\mathrm{CR}} \mathbf{S}_{2}^{\dagger} \mathbf{H}_{\mathrm{CR}}^{\dagger} \mathbf{S}_{1}^{\dagger}\right) \\
\text { subject to } & 0 \leq\left(\mathbf{S}_{i}\right)_{j j} \leq 1, j=1, \ldots, M_{C R} \text { if } i=1 \\
& \text { and } j=1, \ldots, N_{C R} \text { if } i=2 \\
& \operatorname{Tr}\left(\mathbf{Q}_{\mathrm{CR}}\right) \leq P_{C R}, \operatorname{Tr}\left(\mathbf{S}_{1}\right)=m_{c r}, \operatorname{Tr}\left(\mathbf{S}_{2}\right)=n_{c r} \\
& \operatorname{Tr}\left(\mathbf{H}_{\mathrm{INT}} \mathbf{Q}_{\mathrm{CR}} \mathbf{H}_{\mathrm{INT}}^{\dagger} \leq \Omega, \quad \mathbf{Q}_{\mathrm{CR}} \succeq \mathbf{0}\right. \\
\text { variables } & \mathbf{S}_{1}, \mathbf{S}_{2}, \mathbf{Q}_{\mathrm{CR}}(\text { diagonal })
\end{aligned}
$$

We note that the optimization problem $\mathbf{P 2}$ is still not convex (because the objective function is not concave). Here we seek a convex approximation (CA) to this problem. It can be shown that with two of the three variables known, the cost function is concave in the third one and this renders the problem convex in this variable. For example, with $\mathbf{S}_{1}$ and $\mathbf{Q}_{\mathrm{CR}}$ known the cost function is concave in $\mathbf{S}_{2}$ where we rely on a diagonal $\mathbf{Q}_{\mathrm{CR}}$ so that $\mathbf{S}_{\mathbf{2}} \mathbf{Q}_{\mathrm{CR}} \mathbf{S}_{\mathbf{2}}^{\dagger}=\mathbf{U}_{\mathrm{CR}} \mathbf{S}_{\mathbf{2}} \mathbf{U}_{\mathrm{CR}}$, where $\mathbf{U}_{\mathrm{CR}}=\left(\mathbf{Q}_{\mathrm{CR}}\right)^{1 / 2}$. Similarly, with $\mathbf{S}_{2}$ and $\mathbf{Q}_{\mathrm{CR}}$ known, the determinant in the objective function can be written as $\operatorname{det}\left(\mathbf{I}_{N_{C R}}+\mathbf{S}_{2}^{\dagger} \mathbf{H}_{\mathrm{CR}}^{\dagger} \mathbf{S}_{1} \mathbf{H}_{\mathrm{CR}} \mathbf{S}_{2}\right)$ which gives a convex problem in $\mathbf{S}_{1}$. Note that the problem cannot be made convex in $\mathbf{S}_{1}$ if $\mathbf{K}$ is also included in the argument of the cost function. Finally, the log-determinant in $\mathbf{P 1}$ already provides a convex problem in $\mathbf{Q}_{\mathrm{CR}}$. Thus to solve $\mathbf{P} 2$, we initialize $\mathbf{S}_{1}, \mathbf{Q}_{\mathrm{CR}}$ and optimize over $\mathbf{S}_{2}$. After obtaining $\mathbf{S}_{2}$, we optimize over $\mathbf{S}_{1}$ and then, with $\mathbf{S}_{2}$ and $\mathbf{S}_{1}$ known, we obtain the optimum value of $\mathbf{Q}_{\mathrm{CR}}$. This procedure is repeated until the rate achieved stabilizes. The indices of the receive and transmit antennas to be selected are then obtained by choosing the largest $m_{c r}$ and $n_{c r}$ diagonal entries of $\mathbf{S}_{1}$ and $\mathbf{S}_{2}$ respectively. After rounding the possibly fractional diagonal entries of $\mathbf{S}_{1}$ and $\mathbf{S}_{2}$, we again optimize over $\mathbf{Q}_{\mathrm{CR}}$. This optimization involves the original interference constraint of $\mathbf{P 1}$ over the selected CR transmit antennas.

A comment on the convergence of the proposed iterative algorithm is in order here. Using a similar approach to [16], we argue that during the $(k+1)$ th iteration we calculate $\mathbf{S}_{2}^{k+1}=$ $\operatorname{argmax}_{\mathbf{S}_{2}} \mathbf{P 2}\left(\mathbf{S}_{1}^{k}, \mathbf{Q}_{\mathrm{CR}}^{k}, \mathbf{S}_{2}\right)$ and obtain data rate $a$. Then we calculate $\mathbf{S}_{1}^{k+1}=\operatorname{argmax}_{\mathbf{S}_{1}} \mathbf{P 2}\left(\mathbf{S}_{1}, \mathbf{Q}_{\mathrm{CR}}^{k}, \mathbf{S}_{2}^{k+1}\right)$ giving rate $b$. Finally, we evaluate $\mathbf{Q}_{\mathrm{CR}}^{k+1}=\operatorname{argmax}_{\mathbf{Q}_{\mathrm{CR}}} \mathbf{P} 2\left(\mathbf{S}_{1}^{k+1}, \mathbf{Q}_{\mathrm{CR}}, \mathbf{S}_{2}^{k+1}\right)$ and the corresponding data rate $c$. Since $a \leq b \leq c$ forms a monotonically increasing sequence which is bounded above (due to input power constraints) we conclude that the sequence of data rates converges to a limit. Our simulations indicate that iterating 6 times for the SU case (and $8-10$ times for the MU case) is almost always sufficient to attain an optimum value of $\mathbf{P 2}$. Since the problem is not convex in nature, the maximum CR rates obtained from $\mathbf{P 2}$ may not be globally optimum. However, results suggest that the values obtained are robust and are globally optimal most of the time for the 
parameters and scenarios discussed in Sec. IV.

\section{Heuristic}

From the above discussion it is evident that apart from being cumbersome, the $\mathrm{CA}$ approach suffers from various drawbacks. For example, complexity depends on the efficiency of the convex optimizer and the number of iterations needed to reach the optimal point. Also, the approach cannot be used with a full $\mathrm{Q}_{\mathrm{CR}}$ matrix or in the presence of interference. To overcome these problems, we propose a heuristic involving norm-based transmit and receive antenna selection [6]-[8]. Norm based selection for i.i.d. channels with no interference constraints is straightforward and involves selecting the rows and columns of the channel matrix with the highest norms. In our situation the interference constraint prescribes different allowable powers for each transmit antenna and the interference plus noise covariance matrix results in different correlations for different receive antenna selections. Hence, selection at both TX and RX is more complex and any approach must handle these difficulties. At the RX end we proceed by selecting the rows of $\mathbf{K}^{-1 / 2} \mathbf{H}_{\mathrm{CR}}$ with the highest norm. This approximates the effect of $\overline{\mathbf{K}}$ without the need to cycle through the possible RX antenna selections. At the TX end the total transmit power is limited to $P_{C R}$ with no constraints per antenna. In the heuristic it is simpler to assume that the maximum available transmit power from any CR TX antenna is bounded by $P_{C R}$. The idea behind the per-antenna power constraint is that antenna A is likely to be more effective than antenna $B$ if, when they are both allocated maximum power, antenna $\mathrm{A}$ has a higher norm under interference constraints. The power inflation intrinsic to this approach is not a problem since we are solely ranking antennas at this stage. After selection the correct power allocation is performed via the $\mathbf{Q}_{\mathrm{CR}}$ matrix. Note that the version of this algorithm described below deals with the MU case only, since this is the more complex case. A similar heuristic for the SU case follows in a straightforward way. Also, we assume that $\omega_{1}=\ldots=\omega_{M_{P U}}=\omega$ for all $M_{P U}$ single antenna PUs. The heuristic is given by:

1) Calculate $P_{j}=\min \left\{P_{C R}, \min \left\{\frac{\omega}{\left\|\mathbf{H}_{\mathrm{INT}}(:, j)\right\|_{2}^{2}}\right\}\right\}$, where $j=1,2, \ldots, N_{C R}$.

2) Evaluate $\tilde{\tilde{H}}=\mathbf{K}^{-1 / 2} \mathbf{H}_{\mathrm{CR}} \operatorname{diag}\left(\left[\sqrt{P_{1}}, \ldots, \sqrt{P_{N_{C R}}}\right], 0\right)$.

3) Find the column norms of $\widetilde{\mathbf{H}}$, i.e., $\|\widetilde{\mathbf{H}}(:, j)\|_{2}^{2}, j=$ $1,2, \ldots, N_{C R}$.

4) Obtain the matrix $\widetilde{\mathbf{H}}_{\mathrm{COL}}$ by keeping the $n_{c r}$ columns with the highest norms and setting the remaining $N_{C R}-$ $n_{c r}$ columns of $\widetilde{\mathbf{H}}$ equal to zero. This gives $\mathbf{S}_{2}$.

5) Determine the top $m_{c r}$ rows of $\widetilde{\mathbf{H}}_{\mathrm{COL}}$ on the basis of the row norms, $\left\|\widetilde{\mathbf{H}}_{\mathrm{COL}}(k,:)\right\|_{2}^{2}, k=1,2, \ldots, M_{C R}$. This gives $\mathbf{S}_{1}$.

6) With the final selection, $\mathbf{S}_{1}$ and $\mathbf{S}_{2}$, optimize the achievable rate over $\mathbf{Q}_{\mathrm{CR}}$ subject to $\operatorname{Diag}\left(\mathbf{H}_{\mathrm{INT}} \mathbf{S}_{2} \mathbf{Q}_{\mathrm{CR}} \mathbf{S}_{2}^{\dagger} \mathbf{H}_{\mathrm{INT}}^{\dagger}\right) \leq(\omega, \ldots, \omega)$ and the total transmit power constraint.

It is worth noting that the above heuristic can be optimized with a full $\mathbf{Q}_{\mathrm{CR}}$ matrix and is not restricted to a diagonal form as in the CA approach.

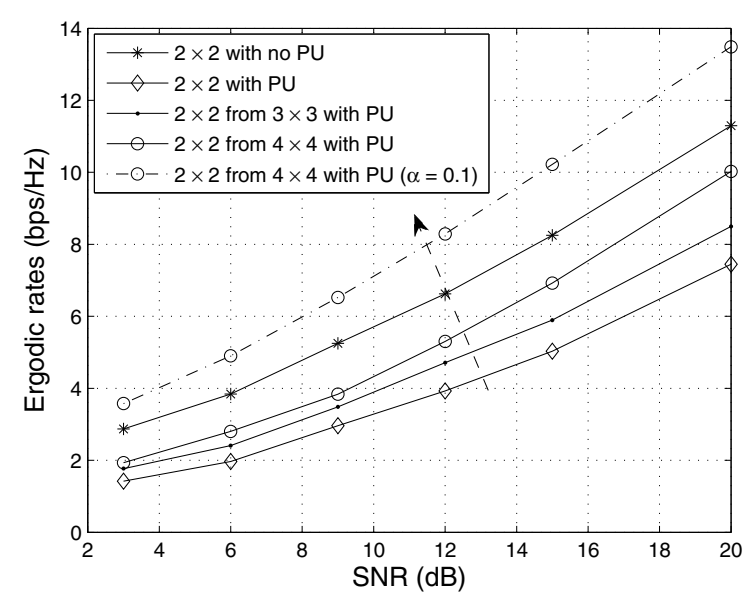

Fig. 2. Ergodic rates vs SNR for different system sizes. These curves are based on the CA approach for the SU case. For all curves (except the one indicated in the figure) we take $\alpha=0.5$ and $\beta=0.1$.

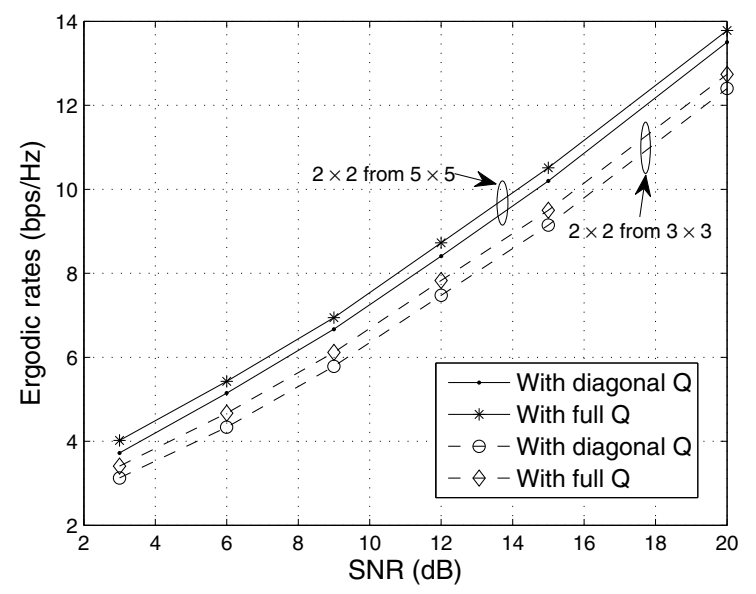

Fig. 3. Ergodic rates vs SNR for different system sizes and in the presence of a PU TX with 3 antennas. $\alpha=0.5$ for both from CR TX to PU RXs and PU TX to CR RX. We take $\beta=0.35$. Antenna selection is performed using the heuristic and both diagonal and full $\mathbf{Q}_{\mathrm{CR}}$ matrices are considered.

The heuristic is an extension of the simple norm-based criteria [6]-[8] with interference constraints added. We stress that the per antenna power-constraint is not real but is used to avoid any iteration over antenna power allocation. This makes the heuristic able to select antennas based solely on row and column norms which is much faster to compute.

\section{RESULTS}

In this section we explain the simulation results based on the ES, CA and the heuristic proposed in Sec. III. However, before we elaborate the results, we introduce the parameter $\beta$ which controls the interference threshold $(\Omega$ for the SU case and $\omega_{j}, j=1, \ldots, M_{P U}$ for the MU case) at the PU RX. $\beta$ is chosen so that the allowable interference at the PU is a fraction of the PU SNR, i.e., $\Omega$ or $\omega_{j}=\beta \mathrm{SNR}_{\mathrm{PU}}$ at the PU RX(s). To compare the different approaches we use the measures of ergodic rates and the cumulative distribution function (CDF) of the achievable rates. The CDF curves and each point on 


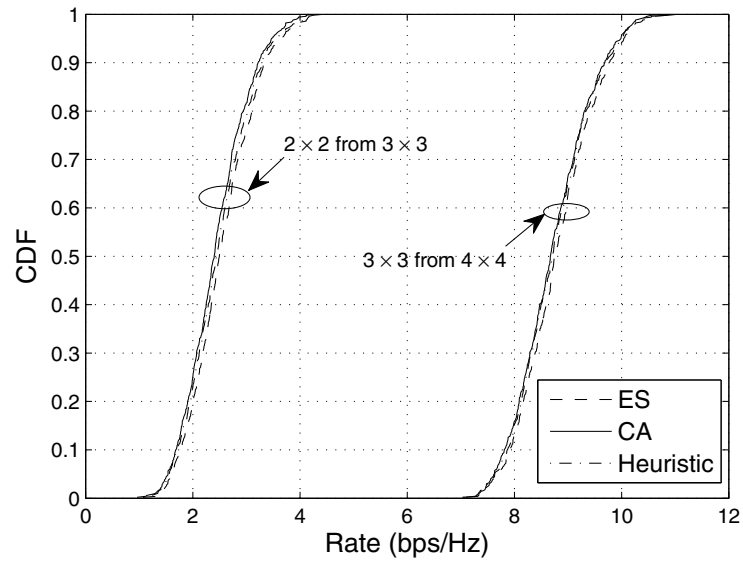

Fig. 4. CDFs of rates achieved for various selection methods for two different systems at $\mathrm{SNR}=8 \mathrm{~dB}$ for the MU case with 3 single antenna PUs.

the ergodic rate graphs are determined by averaging over the results obtained from 500 i.i.d. channel realizations. For the SU case we consider a single MIMO PU RX equipped with 3 antennas and for the MU case we take three PUs each having a single antenna (Fig. 1). The results shown focus on the rate gains offered by selection, the effects of diagonal $\mathbf{Q}_{\mathrm{CR}}$ (important in the CA approach) and a comparison of the techniques. For reasons of space a comprehensive study is not possible and this is left to an extended journal version of the paper.

In Fig. 2 we demonstrate that $\left(n_{c r}, m_{c r}\right)$ antenna selection from larger $\left(N_{C R}, M_{C R}\right)$ systems can enhance the ergodic rates to reach and go beyond the benchmark performance of an $\left(n_{c r}, m_{c r}\right)$ system without any PU interference constraints. These graphs are based on the SU case and are obtained using the CA approach for diagonal $\mathbf{Q}_{\mathrm{CR}}$. In particular, we see that if we select the best $(2,2)$ antenna subsystem (according to P2) from $(3,3)$ and then from $(4,4)$ MIMO channel matrices, we are able to close the gap between the ergodic curves for these systems and the results for the benchmark $(2,2)$ MIMO system without any PU. These results are for $\alpha=0.5$ and $\beta=$ 0.1 . Further, if the strength of the CR-PU interference channel is lowered with respect to the CR-CR channel by decreasing $\alpha$ from 0.5 to 0.1 (which is plausible for environments with shorter range CRs), the ergodic rate curve for a $(2,2)$ system obtained from a $(4,4)$ system goes beyond that of a $(2,2)$ system without any PU operating in its vicinity. This clearly indicates that even after performing antenna selection subject to the interference constraints, there are still enough degrees of freedom left for the CR to attain a substantial gain in terms of its maximum achievable rates.

In Fig. 3 we consider the effects of a diagonal input covariance matrix and incorporate interference from the PU TX. We plot the ergodic rate curves based on the heuristic for two different systems and make a comparison between a diagonal and a full $\mathbf{Q}_{\mathrm{CR}}$ matrix in the presence of an interfering PU TX. For reasons of symmetry and to avoid any further parameters we assume that the signal strength from the PU TX to the CR RX is also given by the parameter $\alpha$. Hence, each element of $\mathbf{H}_{\mathrm{PC}}$ has power equal to $\alpha$. The results are shown in Fig. 3. For these results we considered a CR device with three single antenna PU RXs in its vicinity and a PU TX equipped with 3 antennas interfering at the CR RX. As expected there is a small loss of rate for both systems when $\mathbf{Q}_{\mathrm{CR}}$ is restricted to diagonal form. However, the rate loss for the larger system is slightly less than that of the smaller one.

In the absence of PU-CR interference all 3 techniques can be used and their performance is compared in Fig. 4 via CDFs of the achievable rates. The three techniques follow a hierarchy of complexity from the full solution in the ES through the relaxed iterative optimization in CA to the simple heuristic. Hence, it is notable that all 3 methods are remarkably similar and that even with its massively reduced complexity the heuristic is very similar to the CA approach and only a little behind the ES. Although the relative performance needs to be investigated in more detail over a wider range of parameters and scenarios, this is an excellent indication that near optimal results may be achieved with a very simple selection heuristic.

As a final remark we compare the computational complexity of the heuristic and the CA methods. As mentioned above, the ES method is not practically feasible so is not considered. Although, the CA approach is based on evaluating a series of convex programs which can be efficiently solved using polynomial time interior point methods [13], its complexity heavily depends on the number of iterations required to attain a stable value. In contrast, the heuristic only involves a single such convex optimization problem. Thus on our workstations, the heuristic was found to be approximately $6-8$ times quicker than the CA approach, for systems with up to $5 \mathrm{TX}$ or RX antennas.

\section{CONCLUSION}

In this paper we have used the idea of antenna selection to jointly satisfy interference constraints at the PU while improving the achievable rates of the CR device. We have presented three schemes in order of decreasing complexity to solve this problem. The optimal search approach is the most computationally intensive while the CA approach solves the problem at hand by iteratively optimizing a series of small convex programs. We then present a norm-based separate transmit receive antenna selection technique. This approach results in huge complexity reductions and produces very accurate results. It is notable that this simple technique performs almost indistinguishably from the CA approach which is a well established optimization approach to approximating the full solution. Finally, we have shown that antenna selection can lead to performance improvements for MIMO CR devices even under interference constraints.

\section{ACKNOWLEDGEMENT}

The authors wish to acknowledge the helpful advice of Dr. Michael C. Grant on using CVX. They also wish to extend their gratitude to Prof. Desmond P. Taylor for pointing out the issue of convergence in the CA approach and the anonymous reviewers whose helpful comments greatly improved the presentation of the paper. 


\section{REFERENCES}

[1] IEEE P802.22/D0.1 Draft Standard for Wireless Regional Area Networks, 802.22 Working Group Std., May 2006. [Online]. Available: http://grouper.ieee.org/groups/802/22/

[2] G. Scutari, D. Palomar, and S. Barbarossa, "Cognitive MIMO radio," IEEE Signal Processing Magazine, vol. 25, no. 6, pp. 46-59, Nov. 2008.

[3] R. Zhang and Y. C. Liang, "Exploiting multi-antennas for opportunistic spectrum sharing in cognitive radio networks," IEEE Journal of Selected Topics in Signal Processing, vol. 2, no. 1, pp. 88-102, Feb. 2008.

[4] L. Zhang, Y. Xin, and Y. C. Liang, "Weighted sum rate optimization for cognitive radio MIMO broadcast channels," in Proc. IEEE Conference on Communications (ICC), May 2008, pp. 3679-3683.

[5] S. Sridharan and S. Vishwanath, "On the capacity of a class of MIMO cognitive radios," IEEE Journal of Selected Topics in Signal Processing, vol. 2, no. 1, pp. 103-117, Feb. 2008.

[6] A. F. Molisch and M. Z. Win, "MIMO systems with antenna selection," IEEE Microwave Magazine, vol. 5, no. 1, pp. 46-56, Mar. 2004.

[7] S. Sanayei and A. Nosratinia, "Antenna selection in MIMO systems," IEEE Communications Magazine, vol. 42, no. 10, pp. 68-73, Oct. 2004.

[8] P. J. Smith, T. W. King, L. M. Garth, and M. Dohler, "A power scaling analysis of norm-based antenna selection techniques," IEEE Transactions on Wireless Communications, vol. 7, no. 8, pp. 3140-3149, 2008.

[9] J. Zhou, J. Thompson, and I. Krikidis, "Multiple antennas selection for linear precoding MISO cognitive radio," in Proc. IEEE Wireless
Communications and Networking Conference (WCNC), Apr. 2009, pp. $1-6$.

[10] H. Gao, P. J. Smith, and M. V. Clark, "Theoretical reliability of MMSE linear diversity combining in rayleigh-fading additive interference channels," IEEE Transactions on Communications, vol. 46, no. 5, pp. 666672, May 1998.

[11] M. Kang, L. Yang, and M. S. Alouini, "Capacity of MIMO rician channels with multiple correlated rayleigh co-channel interferers," in Proc. IEEE Global Telecommunications Conference (GLOBECOM), Dec. 2003, pp. 1119-1123.

[12] A. Dua, K. Medepalli, and A. J. Paulraj, "Receive antenna selection in MIMO systems using convex optimization," IEEE Transactions on Wireless Communications, vol. 5, no. 9, pp. 2353-2357, Sep. 2006.

[13] S. Boyd and L. Vandenberghe, Convex Optimization. Cambridge University Press, 2004.

[14] M. Grant and S. Boyd, CVX: Matlab software for disciplined convex programming, June 2009. [Online]. Available: http://stanford. $\mathrm{edu} / \sim \mathrm{boyd} / \mathrm{cvx}$

[15] D. P. Bertsekas, Convex Optimization Theory. Nashua: Athena Scientific, 2009.

[16] P. Ubaidulla and A. Chockalingam, "Non-linear transceiver designs with imperfect CSIT using convex optimization," in Proc. IEEE Wireless Communications and Networking Conference (WCNC), Apr. 2009, pp. $1-6$. 